\title{
The Design Study of the Target Station for the ESS Neutrino Super Beam Project
}

\author{
Loris D'Alessi ${ }^{* \dagger}$ \\ IPHC, Université de Strasbourg, CNRS/IN2P3 \\ F-67037 Strasbourg, France \\ E-mail: loris.dalessieiphe.cnrs.fr
}

\begin{abstract}
The ESS $v$ SB project proposes the production of a European neutrino Super Beam for the discovery of the CP symmetry violation in the leptonic sector. For this purpose, an upgrade is under design of the $5 \mathrm{MW}, 2 \mathrm{GeV}$ proton beam from the LINAC of the European Spallation Source, currently under construction in Lund (Sweden), to obtain an additional $5 \mathrm{MW}$ power beam dedicated to the neutrino production, without therefore reducing the neutron production efficiency. The additional proton beam will be then directed to an accumulator and split through a switchyard in four beams, each of $1.25 \mathrm{MW}$ power. The secondary beam will be delivered to a target station consisting of four target-horn collectors, a decay tunnel, and a beam dump. A dedicated design study of the Target Station is required to optimize the lifetime of the horn, regarding mechanical and thermal constraints, and the $\mathrm{CP}$ sensitivity of the experiment. The current status of this design study is here presented. This project is supported by a COST Action and an EU H2020 Design Study.
\end{abstract}

The 21st international workshop on neutrinos from accelerators (NuFact2019)

August 26 - August 31, 2019

Daegu, Korea

\footnotetext{
${ }^{*}$ Speaker.

${ }^{\dagger}$ On behalf of the ESS $v$ SB Collaboration
} 


\section{Introduction}

Since the measurement of the relatively high value of the neutrino mixing angle $\theta_{13}$, several experiments have been proposed for the precise measurement of the neutrino CP-violating phase $\delta_{C P}$, such as DUNE [1] and T2HK [2]. Recently, the T2K experiment reported the observation of possible hints of a non-null CP-violating phase, the results of the measurements showing a preference for a maximal $\mathrm{CP}$-violation, with the $\mathrm{CP}$ conserving values excluded at $2 \sigma$ confidence level [3]. A European project is currently under design study, namely the European Spallation Source Neutrino Super Beam (ESSvSB) [4], which will take advantage of the high intensity proton beam from the European Spallation Source (ESS) facility [5], currently under construction in Lund (Sweden), for experiments of precise measurements in the neutrino oscillation field. In particular, the expected high intensity of the neutrino beam which could be produced would allow to increase the precision in the measurement of the $\delta_{C P}$ phase. The far detector will be located around the second maximum of neutrino oscillation. In this case, the reduced statistics compared with the other experiments, for which the detection will occur at the first maximum, will be compensated by a reduced importance of systematics errors. The design of the target station for this project is particularly important for defining the characteristics of the neutrino beam. The current status of this design is reviewed in the next sections.

\section{The ESS $v$ SB Project}

The ESS proton linac will be able to deliver protons at $2 \mathrm{GeV}$ kinetic energy, in pulses of 5 MW average power each, with $2.86 \mathrm{~ms}$ duration and $14 \mathrm{~Hz}$ repetition rate. The resulting duty cycle of the linac will be therefore $4 \%$. The linac can be upgraded up to a proton kinetic energy of 3.5 $\mathrm{GeV}$.

By doubling the repetition rate, another proton beam can be obtained which can be used for the production of neutrino beams. In order to improve the performance of the neutrino experiments, the proton kinetic energy required for the $\mathrm{ESS} v \mathrm{SB}$ project is $2.5 \mathrm{GeV}$. The protons extracted will be then directed towards the target station, where they interact with a solid target to produce mesons, in particular pions which, after been focused by the magnetic field produced in a magnetic horn, are directed towards a decay tunnel where they decay to produce neutrinos.

Due to the high intensity current in the magnetic horn necessary to produce the required magnetic field, the proton pulse needs to be shortened to order of $1 \mu \mathrm{s}$. This requires the construction of an accumulator ring. A first estimation of the circumference of the ring gives a value of about 380 $\mathrm{m}$. Since it is difficult to inject protons in the accumulator while other protons are circulating, it has been proposed to accelerate $\mathrm{H}^{-}$ions in the linac and to strip the electrons during the injection by using stripping foils or laser stripping devices. Furthermore, in order to mitigate the effects of the interaction of the high power beam with a solid target, the proton beam will be split in 4 beams of 1.25 MW each after passing through a switchyard located before the target station, and each beam will interact with one of the four target of the four-horn system.

A near and a far detector are then required, the former to monitor the neutrino flux and to measure neutrino interaction cross sections, while the latter will be used for measuring the CP- 

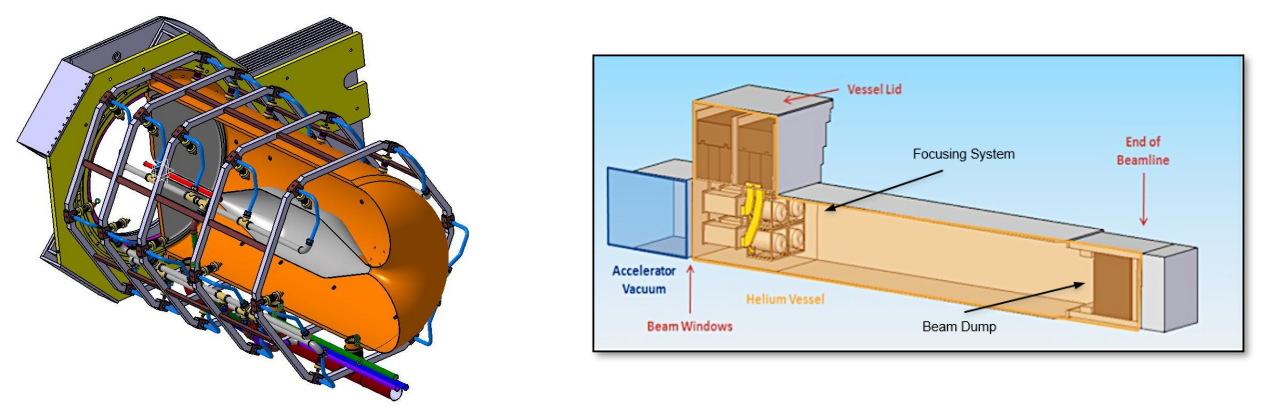

Figure 1: Conceptual design of the magnetic horn (left) and target station layout (right).

violating neutrino phase. The current candidate sites for the far detector are the Galperberg and the Zinkgruvan mine sites, located at $540 \mathrm{~km}$ and $360 \mathrm{~km}$ far from the target station, respectively.

\section{The ESS $v$ SB Target Station}

The design of the target station follows the results of the EUROnu Design Study [6, 7]. The main components of the ESS $v$ SB target station consist of a focusing system, a decay tunnel and a beam dump. The target station chamber is surrounded by a mobile iron shielding supporting the target station. The components of the target station are contained in a vessel filled with helium gas, which will serves also as cooling medium. In Figure 1 the conceptual design of the magnetic horn (left) and of the target station (right) are shown.

The focusing system consists of four magnetic horns, each including a target for the hadron production. The magnetic horn design has a MiniBooNE-like shape, of approximately $60 \mathrm{~cm}$ radius and about $2.5 \mathrm{~m}$ length. The magnetic field is produced by $350 \mathrm{kA}$ pulses with $1 \mu \mathrm{s}$ time width circulating in the horn's skin made of aluminum alloy Al 6061 T6. The proposed target consists of a packed bed of titanium spheres, contained in a canister made of titanium of $78 \mathrm{~cm}$ length and $1.5 \mathrm{~cm}$ radius. Holes are located in the canister to allow the flow of pressurized helium gas (10 bars) through the packed bed. The decay tunnel of $25 \mathrm{~m}$ length, has been designed long enough to allow the mesons to decay and at the same time not long enough to allow the decay of a significant fraction of muons. The beam dump will be designed for the absorption of the protons and secondaries from the decay tunnel. It will consist of graphite block, occupying a volume of $4 \times 4 \times 3.5 \mathrm{~m}^{3}$. Studies have been carried out on the energy deposited in the various components of the target station [8]. According to the reported results of these studies, the total energy deposited in each target is $168 \mathrm{~kW}$, while for each horn the power deposited is of $50 \mathrm{~kW}$. The total power dissipated in the layout is $4.22 \mathrm{MW}$.

\section{Neutrino Production in the Target Station}

The production of secondary particles has been simulated with FLUKA [9, 10], in which the entire experimental layout geometry has been implemented. The same code is used also for energy deposition and activation studies. The current design of the target station is dedicated to the optimization of the neutrino production. The neutrino flux produced by considering for the proton 

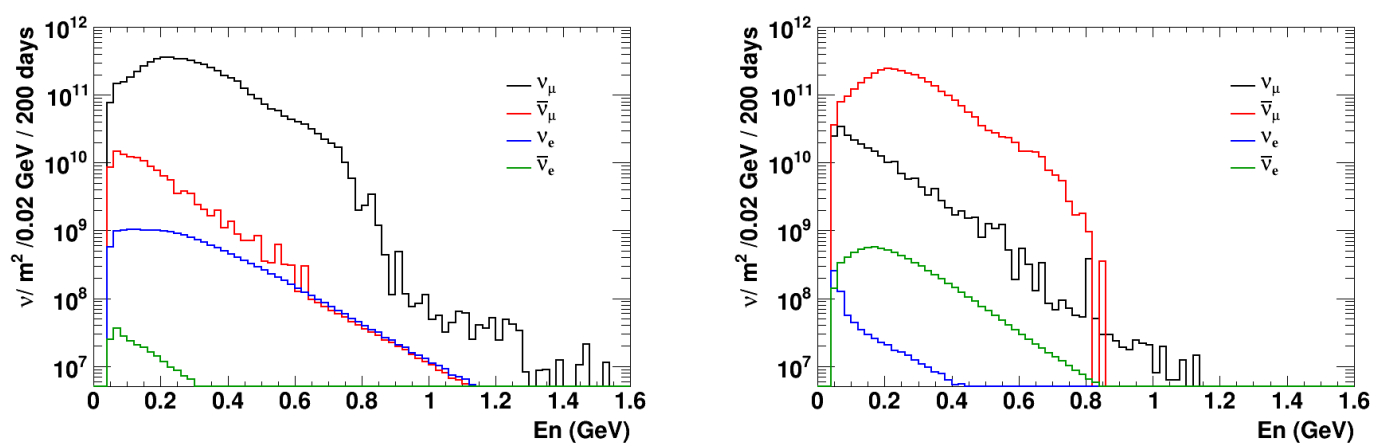

Figure 2: Neutrino flux for positive (left) and negative (right) polarity of the current in the magnetic horn. The flux is calculated at the arbitrary distance of $100 \mathrm{~km}$ from the target station.

beam a gaussian profile of $1 \sigma=4 \mathrm{~mm}$ and $2.5 \mathrm{GeV}$ kinetic energy are shown in Figure 2, both for positive and negative polarity of the current passing in the magnetic horn, and calculated at the arbitrary distance of $100 \mathrm{~km}$ from the target station.

The main contribution to the neutrino flux comes from neutrinos produced by pion decay, while the contribution from the electron neutrinos, mainly due to the muon decay, represents about $0.5 \%$ of the total flux.

\section{Extraction of Muons from the Beam Dump}

With the current design of the target station, more than $10^{20}$ muons can be collected per year. The muons produced in the decay tunnel and entering the beam dump can be extracted for further $\mathrm{R} \& \mathrm{D}$ on muon physics based accelerators and for projects such as low energy $v$ STORM. It has been shown that the same muons can be also used for the production of Higgs bosons [11]. The kinetic energy distribution of the pions and muons reaching the beam dump is shown in Figure 3. The mean kinetic energies are $0.63 \mathrm{GeV}$ for the pions and $0.38 \mathrm{GeV}$ for the muons.
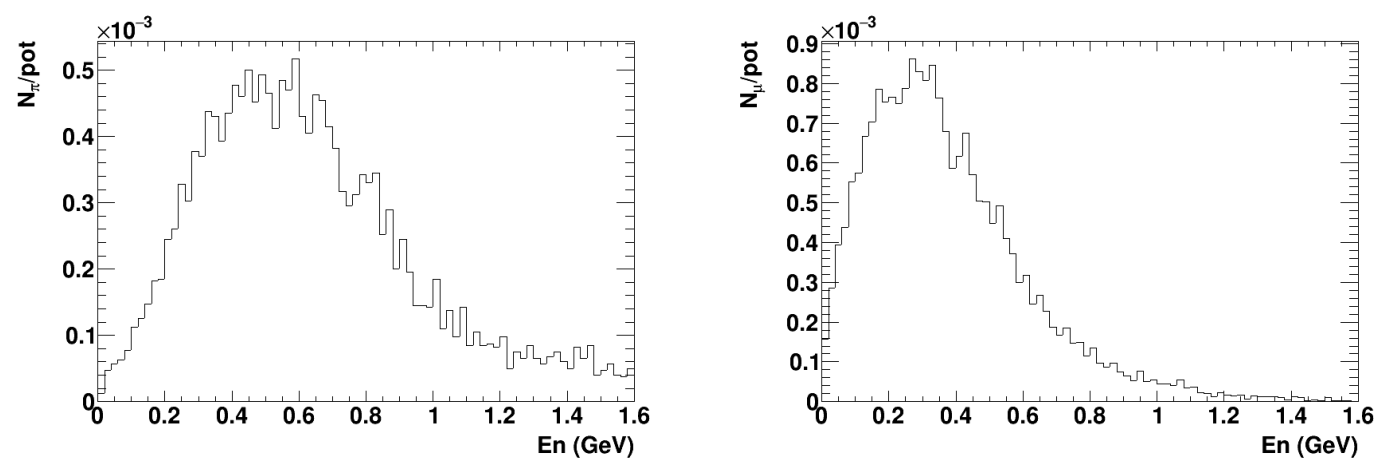

Figure 3: Kinetic energy distribution of pions (left) and muons (right) entering the beam dump.

\section{Conclusion}

The ESS $v$ SB project is currently under design study. The results of this design study will be 
collected in a Conceptual Design Report (CDR) which will be delivered by the end of 2021. The main goal of the project is to provide precise measurement of the $\delta_{C P}$ phase. However, the scientific program can cover a large range of particle physics related subjects, such as neutrino interaction cross sections measurements, muon colliders and Higgs factory. In this framework, the design of the target station plays an important role in the characterization and optimization of the particle production. The design of the whole layout of the target station will be performed following the ALARA (As Low As Reach Achievable) principle and taking into account the safety conditions for the workers and the regulation rules in Sweden.

\section{Acknowledgments}

This project has received funding from the European Union's Horizon 2020 research and innovation programme under grant agreement No 777419.

This project is supported by the COST Action CA15139 "Combining forces for a novel European facility for neutrino-antineutrino symmetry-violation discovery" (EuroNuNet).

\section{References}

[1] DUNE Collaboration, An Experimental Program in Neutrino Physics, Nucleon Decay and Astroparticle Physics Enabled by the Fermilab Long-Baseline Neutrino Facility, Letter of Intent Submitted to the Fermilab PAC, P-1062, Jauary 5, 2015.

[2] K. Abe et al. [Hyper-Kamiokande Proto-Collaboration], PTEP 2015, no. 5, $053 \mathrm{C} 02$ (2015) [arXiv:1502.05199 [hep-ex]].

[3] K. Abe et al. [The T2K Collaboration], arXiv:1910.03887v2 [hep-ex] (2019).

[4] E. Baussan et al., A very intense neutrino super beam experiment for leptonic CP violation discovery based on the European spallation source linac, Nucl. Phys. B 885, 127-149 (2014).

[5] https://europeanspallationsource.se/ .

[6] T. R. Edgecock et al., High intensity neutrino oscillation facilities in Europe, Phys. Rev. ST Accel. Beams 16, 021002 (2013) [arXiv:1305.4067 [physics.acc-ph]].

[7] E. Baussan et al., Neutrino super beam based on a supercondicting proton linac, Phys. Rev. ST Accel. Beams 17, 031001 (2014) [ arXiv:1212.0732 [physics.acc-ph] ].

[8] E. Bouquerel, E. Baussan, M. Dracos, N. Vassilopoulos, Energy Deposition and Activation Studies of the ESSVSB Horn Station, in proceedings of 8th International Particle Accelerator Conference (IPAC 2017) (2017).

[9] A. Ferrari, P. R. Sala, A. Fasso, and J. Ranflt, FLUKA: a multi-particle transport code, CERN-2005-10 (2005), INFN-TC-05-11, SLAC-R-773.

[10] V. Vlachoudis, FLAIR: A Powerful But User Friendly Graphical Interface For FLUKA, in Proc. INt. Conf. on Mathematics, Computational Methods \& Reactor Physics (M\&C 2009), Saratoga Springs, New York, 2009.

[11] C. Rubbia, arXiv:1908.05664v3 [physics.acc-ph] (2019). 